\title{
Research Article \\ Conversation with Diabetic Mellitus II Patients: A Grounded Theory Research
}

\author{
Pir Bux Jokhio ${ }^{1 *}, \mathbb{D}$, Shazia Sultan², Shahida Yaseen Khan ${ }^{3}$, Ghulam Abbas Panhwar ${ }^{4}$, Musrat Fatima ${ }^{5}$, \\ Muhammad Yousif Bhatti ${ }^{6}$ \\ ${ }^{1}$ Institute of Nursing, People's University of Medical and Health Sciences, Nawabshah, Shaheed Benazirabad, Sindh, Pakistan \\ ${ }^{2}$ Department of Physiology, Shaikh Zaid Hospital, Rahim Yar Khan, Pakistan \\ ${ }^{3}$ Advanced Health Sciences, Institute of Nursing, Karachi, Pakistan \\ ${ }^{4}$ Benazir College of Nursing, Shaheed Muhtarma Benazir Bhutto Medical University, Larkana, Pakistan \\ ${ }^{5}$ Peoples Nursing School, Liaqaut University of Medical and Health Sciences, Jamshoro, Pakistan \\ ${ }^{6}$ School of Nursing, Indus Hospital, Karachi, Pakistan
}

\section{ARTICLE INFO}

\section{Article History}

Received 04 November 2020

Accepted 02 January 2021

\section{Keywords}

Diabetic mellitus-II grounded theory

health belief model

management of diabetics

nursing

perceptions

qualitative

\begin{abstract}
The main purpose of this grounded theory research was to explore Diabetic Patients' (DPs) experiences related to their chronic illness in the light of the Health Belief Model (HBM). Diabetic mellitus II is a grave public health concern, and its management is the cornerstone to avoid life-threatening complications. In contemporary research, the role of the patient in the decisionmaking process is essential because the holistic care domain warrants including patients' voices. This research marks the first step in this direction. The sampling strategy used for this study was convenience and snowballing. HBM was used to explore and interpret the DP voices. One-to-one interviews with $11 \mathrm{DPs}$ were the source of data collection. The data were analyzed using the constant comparative analysis method, which is recommended for grounded theory research. The results showed that the main problem of patients is their inability to follow the diet plan accurately owing to financial concerns, social limitation, and a desire to eat more. However, all participants were familiar with the signs and symptoms of the disease. One major observation was the accidental diagnosis of the disease, indicating that proper screening is very necessary to reduce its incidence rate. Nursing must educate patients about the necessity of strictly adhering to a diet plan and taking medications on time. Patients' fears and anxiety related to the disease and its complications must be reduced to improve the quality of life.
\end{abstract}

(ㄷ) 2021 Dr. Sulaiman Al Habib Medical Group. Publishing services by Atlantis Press International B.V. This is an open access article distributed under the CC BY-NC 4.0 license (http://creativecommons.org/licenses/by-nc/4.0/).

\section{INTRODUCTION}

Diabetes Mellitus-II (DM-II) is a major global public health concern and an emerging challenge [1]. The World Health Organization estimated a twofold increase in disease burden during the next 25 years [2]. In Pakistan, the prevalence of DM-II is also very high. In a recently conducted survey, the prevalence of DM-II was estimated at $17 \%$ based on hemoglobin A1C screening [3]. In particular, the prevalence of DM-II is increasing in the adult population because of obesity and sedentariness. The average age for DM-II diagnosis has moved from the 50 s to 40 s [4].

A Diabetic Patient (DP) has to realize that management and control of hyperglycemia is the mainstay to defeat complications and remain healthy [5]. This realization depends on the motivation and willingness of DPs, which are aggravated by the duration of the illness. During this period, DPs develop varying attitudes. Some DPs blame themselves, reasoning that this is because of their inability to control their emotions. Others view DM-II as a gift or punishment

*Corresponding author. Email: pjokhyo@pumhs.edu.pk

Peer review under responsibility of the Dr. Sulaiman Al Habib Medical Group Data availability statement: The data that support the findings of this study are available from the corresponding author [PBJ], upon reasonable request. bestowed by the super power authority (Allah). It was also revealed that DM-II is passed down in the family. Others point to the digestive system, that is, DM-II patients believe that the diet they consumed contained sugar and that the body's inability to metabolize food consequently resulted in DM-II [6]. In another study, participants expressed that they had become anxious and fearful after the diagnosis, and that the disease has weakened them, leaving them unable to perform their daily activities. DPs were fearful because they had witnessed others (family members) suffering from DM-II complications. Lastly, their anxiety increased because they feared losing their jobs and social support [7].

Life with DM-II is not easy for DPs. It is exhausting and difficult physically, mentally, and socially. This extends to certain aspects of their lives, such as medication, diet, and self-management interventions. Patients belonging to low socioeconomic strata preferred to check their blood glucose levels manually. Some patients said that they did not check their blood glucose level regularly because of their inability to buy sticks. However, they also revealed that they rushed to have their blood glucose level checked when the symptoms deteriorated [8]. A DP's feelings determined his/her willingness to deal with the crisis. Confident and motivated patients faced the difficult challenge of hyperglycemia self-management, and were able to perform these activities with the support system 
available around them. Turning off the support system may pose a threat of instability in an already compromised DP-for example, healthy people should avoid forcing a DP to eat sweets [9].

There is a dearth of qualitative research to explore DPs' views/opinions about their experiences related to chronic illness. There were two important reasons to conduct this study. The emerging paradigm of holistic care places emphasis on including the patient in the decision-making process related to disease management, because holistic care is the heart of contemporary nursing care, which emphasizes the need of the patient as a focal point of nursing [10]. Without listening to the patient, the aforementioned holistic care goals cannot be achieved. The best way of listening to the patient is by conducting a qualitative study [11]. This research was based on understanding the life experiences of DPs. The study aimed to explore DPs' experiences related to their chronic illness in light of the Health Belief Model (HBM). The objectives of the study were: (1) to explore perceived benefits and perceived barriers (cues to action) in the language of DPs; and (2) to examine perceived susceptibility, perceived severity, and health motivation (actions) in the language of DPs.

\section{HBM AS THEORETICAL FRAMEWORK}

This framework is an excellent source of motivating people toward positive behavioral change. This framework is extensively used and studied to recognize and appreciate public health issues [11]. There are six concepts upon which this model is built: perceived susceptibility, perceived severity, perceived barriers, perceived benefits, cues to action, and self-efficacy [12]. The HBM is useful to clarify, forecast, and stimulate human behaviors with wavering success. It is supposed through HBM that a belief of the susceptibility (concern) for a problem (disease) will motivate (self-efficacy) an individual for a particular action (cues), irrespective of threats or hardships (perceived barriers) [13].

\section{METHODOLOGY}

This study was grounded theory research, and this methodology is important because it presents a way to capture in-depth perspectives of the situation through the subjective exploration of those who experience the situation. In other words, it is the subjective nature of knowledge through first-hand experiences and viewpoints. It helps us understand an individual's point of view concerning his/her experiences, especially how they deal with and what more they need to balance the situation [11]. The study population consisted of DPs living in the different villages of Shaheed Benazir Abad District of Sindh, Pakistan. The sampling methods for this study were convenience and snowball sampling. The inclusion criterion called for male and female DPs living in different villages, whereas the exclusion criterion was a DP with any type of disability. The sample size for this study was 11 .

The method for data collection was individual interviews. This method was applied because the researchers wanted to interact with the patients to understand their unique experiences. Faceto-face interviews were considered a rich source to "enter" into another person's perspective [14]. There were four open-ended questions: (1) How did your life change when you were diagnosed with diabetes? (2) Is there something that worried (scared) you when you received this diagnosis? (3) What types of problems did you face as a DP? (4) What are the strategies that you adopted to deal with this situation?

All interview notes were maintained, and each interview lasted 30-35 min. Informed consent was obtained after the aims and objectives of the study were explained to the participants. Participation in the study was voluntary, and no money or gifts were offered to the participants. The Ethics Review Committee of Peoples University of Medical \& Health Sciences for Women (Nawabshah, Shaheed Benazirabad) granted approval to the study.

Data were analyzed using constant comparative analysis as recommended in grounded theory research $[11,15]$. The unit of analysis in our study was "living with DM-II." From this main hint, researchers read the interview text several times to make sense of the data and identify the content areas. Then, each content area was supported by finding relevant meaning units and condensed. Lastly, condensed meaning units were abstracted and coded. Table 1 shows the condensed areas, condensing units, and codes $[16,17]$. In this way, the final presentation of the main theme, areas, meaning units, and codes were presented for the final discussion.

\section{RESULTS}

The mean ( \pm standard deviation) age of 11 participants was 48.36 $( \pm 5.59)$ years, and ranged between 40 and 55 years [16]. There were eight $(72.72 \%)$ male and three $(27.27 \%)$ female participants, who were selected from three villages (Bahadur Khan Jokhio, Abdul Rehman Panhwar, and Nabi Bux Magsi) located in Union Council Khadher, Taluka Sakrand, District Shaheed Benazir Abad, Sindh, Pakistan. Six participants had only primary education and the remaining participants did not attend any school (Uneducated). One of the participants was Mualim in a village mosque. The remaining male participants were either farmers or dependent on their family members. All three women participants were married housewives. All participants were on oral tablets to control their hyperglycemia and no one was on insulin. The participants were diagnosed with DM-II at least 5 years ago.

\subsection{Health Belief Model}

\subsubsection{Perceived susceptibility}

According to this construct of perceived susceptibility, out of 11 study respondents, 10 demonstrated knowledge related to causes of developing DM-II. The participants cited obesity, high cholesterol diets, lesser activity, domestic tensions, inability to meet family financial needs, and taking saturated sugar items as the main causes of DM-II. However, most of them failed to connect the association between hypertension and DM-II.

The participants gave different descriptions of DM-II.

Participant 1: The sugar in the blood.

Participant 2: The sugar in the urine.

Participant 10: “I am a 'Muallim ul Quran' for the last 20 years. I became a sugar patient because I use to sit for long times (four to 
Table 1 Participants' responses in connection with HBM constructs

\begin{tabular}{|c|c|c|c|}
\hline S\# & HBM (Constructs) & Themes & Code statements \\
\hline & & Financial and social vulnerability & My first is closed \\
\hline & & Blood transfusions during pregnancy & Felt a lot of weak and doctor advised blood transfusions \\
\hline \multirow[t]{4}{*}{2} & Perceived severity & Hypertension & In tense situations, my heartbeat increases a lot \\
\hline & & Sexual problems (erectile dysfunction) & Reduced sexual drive \\
\hline & & Inflammation of blood vessels & My hands and feet became swollen \\
\hline & & Constipation and skin allergy & As a result of using unreliable medication from 'Hakeems' \\
\hline \multirow[t]{2}{*}{3} & Perceived benefits & The sugar level in control & Potatoes, rice, dates, mango, milk must be eaten in moderate amounts \\
\hline & & Activity and exercise & Makes blood thin and is distributed to the whole body easily \\
\hline \multirow[t]{2}{*}{4} & Perceived barriers & Living away from homes (inappropriate diet plan) & A Mualim ul Quran living away from his home \\
\hline & & Inefficient healthcare system & $\begin{array}{l}\text { Government sector people do not care whereas, in private hospitals, } \\
\text { there is the care with respect }\end{array}$ \\
\hline & & Anxiety, depression & Not able to perform daily activities due to mood changes \\
\hline 6 & Self-efficacy & Unable to use a glucometer & Do not know how to check blood glucose \\
\hline & & Don’t use a glucometer & Results are not reliable \\
\hline
\end{tabular}

five hours in a single sitting) to teach Quran Pak (A Holy Book of Muslims). This long sitting resulted in weight gain. Then, I had frequent urination at night time. Therefore, I became the patient..."

Participant 3: "I became the patient of sugar... (DM-II) when I was second time pregnant about 13 years ago. My doctor advised for two consecutive blood transfusions and...I became the victim. Before that, I was totally fine and healthy."

Participant 9: "Sugar is tension. After the death of my parents (father and mother), I became tense.... (Because...) no one is there to look after me. I am married and my husband is a patient of mental illness" (probably schizophrenia). "My firsts are closed" (a Sindhi proverb used to explain poverty and social vulnerability. "I have no children.... Therefore... It hurts me a lot...and at times... My sugar (level) goes very high."

Participants 3, 4, 7, and 8: "I didn't know that I had developed the sugar..., it was accidentally revealed to me when I visited the doctor (Physician) for seeking medications (for another problem)."

Participant 11: "Sugar is lethargy, no interest in daily activities, kidney pain."

\subsubsection{Perceived severity}

In light of this construct, valuable insights were extracted. The participants were aware of the severity of the disease. The participants reported hypertension, kidney pain, inflammation of various parts of the body (face, feet, etc.), sleep disturbance due to urination during nighttime (nocturia), excessive hunger, tooth pain, and body aches as the main DM symptoms.

Participant 10: "I am a patient of sugar. I have reduced need of sexual desire... (sexual drive due to erectile dysfunction). I feel shy to say my doctor about this problem." (Even at the time of the interview, the participant did not reveal much about the problem of erectile dysfunction.)
Participant 5: "I am married for 25 years and diagnosed as a patient of sugar (DM-II) for 17 years. I can't conceive. I went to use different Hakeems (to get alternative therapies for the conception) and they provided me some medicines but didn't succeed. After taking medicines (provided by the Hakeem), I became constipated. It was a big problem.... As skin rashes appeared. There was a lot of muscle pain. When my husband accompanied me to a doctor, he diagnosed sugar (DM-II)."

\subsubsection{Perceived benefits}

The majority of the participants appreciated the benefits of sugar control. They knew that items made with sugar (saturated) are harmful to DPs.

Participants 4, 6, and 10: "Potatoes, rice, dates, mango, milk increase the sugar (level)."

Participant 1, 3, and 7: "A patient of sugar (DM-II) should not sleep for a long time. S/he must work... The activity of any kind is beneficial to control sugar (level). Going outside and walking is good for (patients). Walking is helpful in blood distribution (it makes the blood thin)."

\subsubsection{Perceived barriers}

This construct explored important insights concerning blood sugar control and escaping complications. DPs explored many barriers during this conversation. Those barriers hindered efforts to control optimal blood sugar level.

Participant 10: "Living away from the home is a very stressful condition. I am living away from my home for many years. I visit my home fortnightly. Here, a DP is helpless to manage a diet plan. I have to depend on what is given to me from a nearby family, because, they have taken responsibility for my food. Therefore, 
I can't make suggestions. I have to remain satisfied with what is provided to me...."

Participants 7, 10, and 9: "I used to go to a private clinic to get sugar (related) treatment. Government hospitals are useless. There comes many patients. Although, there is also a huge number of patients in private clinics. But there is a difference. In Government hospitals, no one cares for you. But, in private clinics, doctors check-up with honesty."

Participants 5, 6, 8-11: "Medicines and doctor visits come with money. Money is a big problem. Therefore, it is difficult to manage your problem, if you lack resources."

Participants 2, 3, and 9: "We, go to visit my relatives, or friends or/ and attend social gatherings, it is difficult to follow the diet plan. You eat...sometimes...intentionally, ...sometimes due to restrictions. You cannot say that I am on a diet plan. You have to take what is available. But sometimes, people around you force you by saying 'Take... it doesn't matter.' ... And you eat. Sometimes, you eat because you cannot control yourself."

\subsubsection{Cues to action}

According to the "cues to action" construct, 10 of 11 participants easily and confidently described excessive urination, excessive hunger, dry mouth, hypertension, anxiety, and depression as signs of increased sugar (hyperglycemia) and a need to seek treatment.

Most of the participants: "I become uncomfortable whenever there is urging to urinate or increased heart beating."

"Whenever there is excessive urination, I understand that the sugar (level) has increased. Sometimes, I go to the doctor. But mostly, stay.... And wait for the sugar to decrees...."

Only two of 11 participants rectified the signs and symptoms of low sugar (level). This means that most of them were not aware of their low blood glucose (hypoglycemia) level; however, all were familiar with the solution of hyperglycemia.

"I am very lethargic and my eyesight (vision) has become unclear (blurred)."

"I don't know the sign and symptoms of low blood glucose, but the doctor has advised me to eat sweets whenever there is low sugar level."

\subsubsection{Self-efficacy}

The last construct, "self-efficacy," also unearthed important information in the context of DM-II management. Most of the participants were familiar with the signs and symptoms of high glucose levels but did not know the way to handle the situation.

"My sugar is in control. I discontinue medication out of fear of low sugar (in blood) level. Someone has told me that medicine causes sugar to drop. Therefore, I am conscious."

"I am a patient of high sugar (level) and it remains elevated (use medicines regularly). The doctor has advised me to switch on injections (Insulin...). I refused out of fear of frequent injections two times a day). I believe.... It is very painful and difficult to manage injections twice a day."
"I feel that I am not able to have satisfactory sex. I consulted my doctor. He advised me to start injections for controlling sugar, but I denied it. I don't like to be on injections."

"I am not able to check my blood sugar level through a glucometer."

"I don't check through a glucometer. People say the results are not reliable."

\section{DISCUSSION}

Diabetes mellitus-II is a major public health problem. It has spread at an astonishing rate. Therefore, the strategies to control its rapid spread and manage the patients (already diagnosed) are top priority [1]. One way to manage the problem is a holistic approach in which patients, nurses, and physicians adopt a shared decision-making strategy to achieve the desired outcomes successfully [9]. One of the ways of involving patients in the decision-making process related to their treatment and care context is listening to them through qualitative research. Qualitative research helps tailor the care according to an individual's unique needs following the context they (the patients) live and experience [11], because qualitative research is a rich source of understanding behaviors, needs, and experiences. The main purpose of qualitative research is to be attentive about an apprehension to practice [18].

This research is an effort to achieve the aforementioned goal. Nurses need to be excellent professionals. This means that not only are they required to generate knowledge through research but also to consume research as evidence to make their practice safe, secure, and beneficial for the patient. Using evidence generated from research makes nurses' practice reliable and effective [19]. By using HBM, useful insights are easy to collect from DM-II patients [20].

In this study, the first construct, "perceived susceptibility," patients indicated obesity, hypertension, high cholesterol diet, and sedentary life as the main causes of DM-II. This showed that DPs possessed good knowledge about the causes of DM-II. Unfortunately, before the diagnosis of DM-II, no one related signs and symptoms (excessive urination, hunger, dry mouth, elevated blood pressure, and anxiety) to the disease. Participants only became aware of the diagnosis (accidentally) when they visited a physician or a healthcare facility. A study revealed that after knowing the diagnosis, patients took it as a challenge and adopted a life termed as "shaping to learn the life (in future)." They saw themselves as a resource to manage ailments. Family support was indicated as vital for achieving desired outcomes. They believed that physicians would recommend effective interventions to improve diabetes-related management. Patients' confidence was enhanced if the recommended intervention worked well [21]. In our study, a unique idea was explored. A pregnant woman believed that "blood transfusions" caused her DM-II. A study showed that an acute surge in serum ferritin and hemoglobin after blood transfusion in thalassemia patients might add to an escalation in insulin secretion and resistance [22]. Contrary to the findings, there is no evidence that blood transfusions caused DM-II in pregnant women [23]. Nevertheless, pregnant women may develop gestational diabetes mellitus (GDM), and careful monitoring of blood glucose levels of pregnant women is essential for both mother and expected baby [24].

The construct "perceived severity" covers four themes: hypertension, erectile dysfunction, constipation, skin allergies, and 
inability to conceive. In a qualitative study conducted in Muscat, Oman, participants experienced and talked about serious DM-II complications. Job loss, financial difficulties, multiple hospital visits to receive DM-II-related treatment, physical and emotional uneasiness, and relationship breakdowns with family members were explored [20]. DM-II is a shadowy disease. It requires broadminded attitudes to the highest degree and self-conscious attitudes. The attitude of accepting DM-II as a normal condition is dangerous and suppressive. It stops patients from initiating curative and caring interventions. A self-conscious attitude considers DM-II as a big problem and a progressive one assists individuals to take self-responsibility and exerts potential influences for change [6].

The construct, "Perceived benefits" yielded the themes of "good sugar control for health," and "activity and exercises." These themes are well supported in different studies. Good DM-II management was labeled as a means to achieve good health, feelings of being energized, and free from symptoms [20]. Good sugar level control was correlated with freedom from complications. Participants believed that taking part in sports will do wonders for the DPs as it increases blood circulations and diversion from anxiety- and depression-producing stimuli [5]. DM-II was associated with developing life-threatening complications. The participants' observations showed that deaths of near ones after developing DM-II complications frightened them. DM-II management was identified as the only option to reduce the chances of complication development and improve future life [7].

The construct "perceived barriers" generated four important themes: "living away from homes," "inefficient healthcare system," "financial problems," and "social gatherings." These themes were found in other related studies. The "living away from homes" theme was identified as a unique barrier in this study. In a study conducted in Muscat, Oman, cost, social gatherings, stigma were identified as major barriers in DM management. Cost of access to healthcare system, cost of medicines, and laboratory tests were cited as major concerns. Participants stated that in the case of restriction of eating sweet dishes, the hosts convinced them to eat by describing their food as of good quality (although it should be noted that quality of food was never an issue in this case). Moreover, the visiting DM-II individual used to eat sweet dishes to tell others that they were not different. Those contextual situations exacerbated DM-II management issues badly [6]. In another study, the DPs were described as fearful in relation to their disease diagnosis, treatment, complication development, and financial concerns impinging the DM management process. They were more worried about the stigmatization issue. All these problems made them confused and mentally unstable. The encouraging component of the research was the expressed desire of DM-II individuals to gain expertise in the subject so they could self-manage their problems [7]. There was a need to initiate activities to train them for this purpose.

The construct "cues to action" carried the themes of "excessive urination, thirst, hunger," "hypertension," and "anxiety"/“depression." It was observed that participants had good knowledge about hyperglycemia and the need to take action to reduce it, but they were not fully aware of hypoglycemic indications. A study conducted in Malaysia showed that DM-II patients performed self-care activities 3-4 days a week. The study considered self-care behavior as essential for glycemic control and complication prevention. The study further endorsed that compliance with a diabetic diet is necessary to achieve the desired outcome. The nurses must conduct follow-up on patient treatment compliance to reduce confusion and facilitate their road to success [25]. Moreover, the patient's motivation is the cornerstone for glycemic control-related activities. When a patient demonstrates a feeling of discomfort, anxiety, and depression, this could mean that the patient had failed to comply with the instructions, thus allowing hyperglycemia to develop. The patient must believe that glycemic control is the only way to reduce the chances of developing complications. Any other reason to control hyperglycemia will be useless because this will dampen the patient's motivation consistently [6].

The last construct, "self-efficacy," produced the themes of "unable to use glucometer" and "don't rely on the readings of glucometer." The first theme, "unable to use glucometer," explains two different sides of the problem: unavailability of the device or the inability to use the device. Unavailability of the device is related to the cost of the device, and test strips and prick needles. The theme "unable to use glucometer" suggests the need to train DPs so they can selfcheck. The evidence supports the idea of healthcare providers' and family members' unabated sustenance and reassurance to achieve compliance objectives efficaciously [6]. The DM-II self-efficacy in the context of knowledge about the disease and its complications and skills related to adherence to using diabetic medications on time with proper dosages and self-care behaviors are vital for achieving glycemic control. Lastly, DPs must be psychologically and socially supported because anxiety decreases the chances of performing self-care behaviors. DPs' anxiety levels must be screened for management to help improve the compliance domain. DM-II individuals must feel empowered to perform the required self-care activities [26].

\section{CONCLUSION}

In this is grounded theory research, we utilized HBM to explore the meaning of life events of DPs. The grounded theory research with a theoretical framework (HBM) is not used with DPs in the Pakistani context; therefore, this is a novel study. However, because the sample size of this study ( 11 participants) is very small, we explored and examined the concept of "living with diabetes mellitus" to explain the unique dimensions of this segment of the society. Moreover, the sample for this study was drawn from three remote villages of a district (Shaheed Benazirabad). These remote village populations rarely get a chance to be studied. The study sample comprises people with no education or only primary level education; therefore, their voices need be heard to make the necessary changes in the current scenario of the Pakistani health system.

The study scrutinized and discovered important hidden aspects of DM-II individuals in their own words. The most important concept uncovered was the patients' inability to recognize the signs and symptoms of DM-II at the earliest stage. This invites policymakers to adopt programs that would properly screen and educate people about the signs and symptoms of this disease so they could learn to manage the problem at a very early stage. Second, individuals with DM-II must be educated and trained for proper medication adherence and self-care behaviors to help control their hyperglycemia. People need to know about DM-II complications and management. They must be educated to discourage them from turning to unreliable sources (e.g., traditional healers) for treatments. In addition, DPs must be trained to check their glucose level by 
themselves. And lastly, social integration is necessary to help this group deal with problems that arise from stigmatization. Measures must be adopted to assist DPs in managing co-related problems such as anxiety and depression. Further qualitative research is warranted to devise interventions that aim to reduce anxiety and depression problems and how to improve the socialization of this cohort.

\section{CONFLICTS OF INTEREST}

The authors declare they have no conflicts of interest.

\section{AUTHORS' CONTRIBUTION}

PBJ, SS and SYK contributed in study conceptualization. PBJ, SS, GAP, MF and MYB contributed in data collection and data analysis. PBJ, SS, SYK and MYB participated in drafting the article. Final draft revision, and writing was carried out by PBJ, SS, SYK and GAP.

\section{FUNDING}

This research did not receive any specific grant from funding agencies in the public, commercial, or not-for-profit sectors.

\section{ACKNOWLEDGMENTS}

The authors are grateful to the research participants for their valuable contribution to the research.

\section{REFERENCES}

[1] Hussain A, Ali I. Diabetes mellitus in Pakistan: a major public health concern. Arch Pharm Pract 2016;7;30-2.

[2] Akhter J. The burden of diabetes in Pakistan: the national diabetes survey. J Pak Med Assoc 1999;49;205-6.

[3] Aamir AH, Ul-Haq Z, Mahar SA, Qureshi FM, Ahmad I, Jawa A, et al. Diabetes Prevalence Survey of Pakistan (DPS-PAK): prevalence of type 2 diabetes mellitus and prediabetes using HbA1c: a population-based survey from Pakistan. BMJ Open 2019;9;e025300.

[4] Qureshi SS, Nasir S, Amer WM, Shakir H, Khan GM. An evolving epidemic in younger population - diabetes. Pak J Med Health Sci 2014;8;310-13.

[5] du Pon E, Wildeboer AT, van Dooren AA, Bilo HJG, Kleefstra N, van Dulmen S. Active participation of patients with type 2 diabetes in consultations with their primary care practice nurses - what helps and what hinders: a qualitative study. BMC Health Serv Res 2019;19;814.

[6] Abolghasemi R, Sedaghat M. The patient's attitude toward type 2 diabetes mellitus, a qualitative study. J Relig Health 2015;54; 1191-205.

[7] Papaspurou M, Laschou VC, Partsiopoulou P, Fradelos EC, Kleisiaris CF, Kalota MA, et al. Fears and health needs of patients with diabetes: a qualitative research in rural population. Med Arch 2015;69;190-5.

[8] Gillani SW, Sulaiman SAS, Abdul MIM, Saad SY. A qualitative study to explore the perception and behavior of patients towards diabetes management with a physical disability. Diabetol Metab Syndr 2017;9;58.

[9] Engström MS, Leksell J, Johansson UB, Gudbjörnsdottir S. What is important for you? A qualitative interview study of living with diabetes and experiences of diabetes care to establish a basis for a tailored Patient-Reported Outcome Measure for the Swedish National Diabetes Register. BMJ Open 2016;6;e010249.

[10] Zamanzadeh V, Jasemi M, Valizadeh L, Keogh B, Taleghani F. Effective factors in providing holistic care: a qualitative study. Indian J Palliat Care 2015;21;214-24.

[11] Squires A, Dorsen C. Qualitative research in nursing and health professions regulation. J Nurs Regul 2018;9;15-26.

[12] Jones CL, Jensen JD, Scherr CL, Brown NR, Christy K, Weaver J. The health belief model as an explanatory framework in communication research: exploring parallel, serial, and moderated mediation. Health Commun 2015;30;566-76.

[13] Myneni S, Cobb N, Cohen T. In pursuit of theoretical ground in behavior change support systems: analysis of peer-to-peer communication in a health-related online community. J Med Internet Res 2016;18;e28.

[14] Rosenstock IM, Strecher VJ, Becker MH. Social learning theory and the health belief model. Health Educ Q 1988;15;175-83.

[15] Moser A, van der Bruggen H, Widdershoven G, Spreeuwenberg C. Self-management of type 2 diabetes mellitus: a qualitative investigation from the perspective of participants in a nurse-led, shared-care program in the Netherlands. BMC Public Health 2008;8;91.

[16] Oltmann SM. Qualitative interviews: a methodological discuswsion of the interviewer and respondent contexts. Forum Qual Soc Res 2016;17;1.

[17] Graneheim UH, Lundman B. Qualitative content analysis in nursing research: concepts, procedures, and measures to achieve trustworthiness. Nurse Educ Today 2004;24;105-12.

[18] Miller WR. Qualitative research findings as evidence: utility in nursing practice. Clin Nurse Spec 2010;24;191-3.

[19] Hall A. Qualitative research and its role in nursing knowledge. Nurs Times 2006;102;32-5.

[20] Al Subhi L, Kendall P, Al-Shafaee M, Al-Adawi S. Health beliefs of people with type 2 diabetes in primary health care in Muscat, Oman: a qualitative approach. Int J Clin Nutr Diet 2016;2;106.

[21] Fink A, Fach EM, Schröder SL. 'Learning to shape life'-a qualitative study on the challenges posed by a diagnosis of diabetes mellitus type 2. Int J Equity Health 2019;18;19.

[22] Wankanit S, Chuansumrit A, Poomthavorn P, Khlairit P, Pongratanakul S, Mahachoklertwattana P. Acute effects of blood transfusion on insulin sensitivity and pancreatic $\beta$-cell function in children with $\beta$-thalassemia/hemoglobin $\mathrm{E}$ disease. J Clin Res Pediatr Endocrinol 2018;10;1-7.

[23] Chowdhury N. Diabetes mellitus in the context of blood transfusion. J Pak Med Assoc 2017;67;480-1.

[24] Abourawi FI. Diabetes mellitus and pregnancy. Libyan J Med 2006;1;28-41.

[25] Yee KC, Said SM, Manaf RA. Identifying self-care behavior and its predictors among type 2 diabetes mellitus patients at a district of Northern Peninsular Malaysia. Mal J Med Health Sci 2018;14;17-29.

[26] Tohid H, Papo M, Ahmad S, Sumeh AS, Jamil TR, Hamzah Z. Self-care activities among patients with type 2 diabetes mellitus in Penampang, Sabah, and its association with depression, anxiety, and stress. Mal J Public Health Med 2019;19;117-25. 